\title{
Angiogenic profile in the Finnish Genetics of Pre-Eclampsia Consortium (FINNPEC) cohort
}

\author{
Tiina Jääskeläinen $^{\mathrm{a}, *}$, Seppo Heinonen ${ }^{\mathrm{b}}$, Esa Hämäläinen ${ }^{\mathrm{c}}$, Kari Pulkki ${ }^{\mathrm{d}, \mathrm{e}}$, Jarkko Romppanen ${ }^{\mathrm{d}}$, \\ Hannele Laivuori $^{\mathrm{a}, \mathrm{f}, \mathrm{g}, \mathrm{h}}$, for the FINNPEC ${ }^{1}$ \\ ${ }^{a}$ Medical and Clinical Genetics, University of Helsinki and Helsinki University Hospital, Helsinki, Finland \\ b Obstetrics and Gynecology, University of Helsinki and Helsinki University Hospital, Helsinki, Finland \\ ${ }^{\mathrm{c}}$ Department of Clinical Chemistry, University of Helsinki, Helsinki, Finland \\ d Eastern Finland Laboratory Centre, Kuopio, Finland \\ e Saske Newborn Screening Center, Turku University Hospital, Turku, Finland \\ ${ }^{\mathrm{f}}$ Institute for Molecular Medicine Finland, HiLIFE Unit, University of Helsinki, Helsinki, Finland \\ ${ }^{g}$ Faculty of Medicine and Life Sciences, University of Tampere, Tampere, Finland \\ ${ }^{\mathrm{h}}$ Department of Obstetrics and Gynecology, Tampere University Hospital, Tampere, Finland
}

\section{A R T I C L E I N F O}

\section{Keywords:}

Angiogenic markers

Pre-eclampsia

Soluble fms-like tyrosine kinase 1 (sFlt1)

Placental growth factor (PIGF)

Soluble endoglin (sEng)

\begin{abstract}
A B S T R A C T
Objectives: To study first and second/third trimester levels of soluble fms-like tyrosine kinase 1 (sFlt1), placental growth factor (PIGF) and soluble endoglin (sEng) in FINNPEC case-control cohort. The participants were further divided into subgroups based on parity and onset of the disease. Recommended cut-off values in aid of preeclampsia (PE) prediction and diagnosis were also tested.

Methods: First trimester serum samples were available from 221 women who later developed PE and 239 women who did not develop PE. Second/third trimester serum samples were available from 175 PE and 55 non-PE women. sFlt-1 and PIGF were measured electro-chemiluminescence immunoassays and sEng by ELISA.

Results: In all timepoints PlGF, endoglin and the sFlt-1/PlGF ratio were increased in the PE group compared to the non-PE group. The serum concentrations of sFlt-1 were increased only at second/third trimester in PE women. Higher concentrations of s-Flt1, endoglin and higher sFlt/PlGF ratio were found at the third trimester in primiparous women compared to multiparous women. Primiparous PE women also had lower concentrations of PlGF at the third trimester. The proportion of women exceeding all cut-offs of the sFlt-1/PlGF ratio ( $\geq 33$, $\geq 38$, $\geq 85$ and $\geq 110$ ) was greater in the PE group, but there were also pre-eclamptic women who met rule-out cut-off or did not meet rule-in cut-off.

Conclusions: Primiparous pregnancies have more anti-angiogenic profile during second/third trimester compared with multiparous pregnancies. Our findings also suggest that certain maternal characteristics, e.g. BMI, smoking and pre-existing diseases, should be taken into account when different sFlt-1/PlGF ratio cut-offs are utilized.
\end{abstract}

\section{Introduction}

Pre-eclampsia (PE) is a complex pregnancy disorder, defined by new-onset hypertension and proteinuria after 20 weeks of gestation, or new onset preeclampsia-associated signs in the absence of proteinuria
[1]. Currently there is no treatment for PE other than delivery, which often leads to premature birth due to indicated delivery. As one of the major conditions causing maternal and fetal morbidity and mortality, it is a global challenge for maternal and fetal health care providers [2]. Due to incomplete understanding of the pathogenesis and subtypes of

\footnotetext{
* Corresponding author at: Faculty of Medicine, Department of Medical and Clinical Genetics, Biomedicum P.O. Box 63, FI 00014, University of Helsinki, Finland.

E-mail address: tiina.j.jaaskelainen@helsinki.fi (T. Jääskeläinen).

${ }^{1}$ The FINNPEC core investigator group: Hannele Laivuori (PI) (Medical and Clinical Genetics, University of Helsinki and Helsinki University Hospital, Helsinki, Finland; Institute for

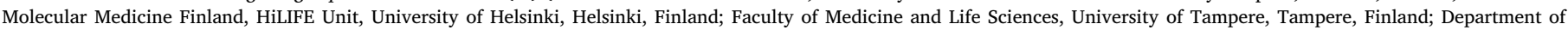

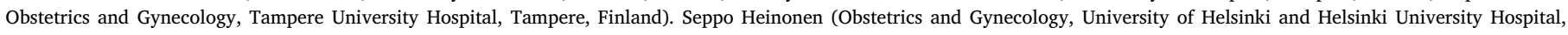

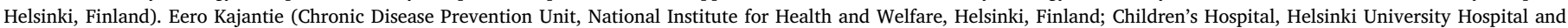

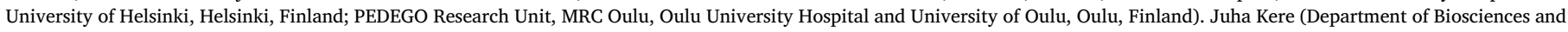

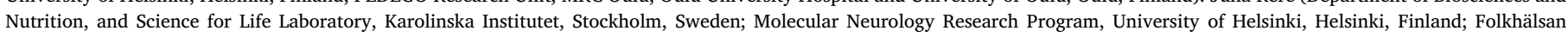

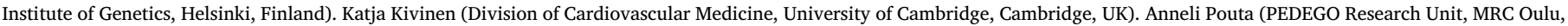
Oulu University Hospital and University of Oulu, Oulu, Finland; Department of Government Services, National Institute for Health and Welfare, Helsinki, Finland).
} 


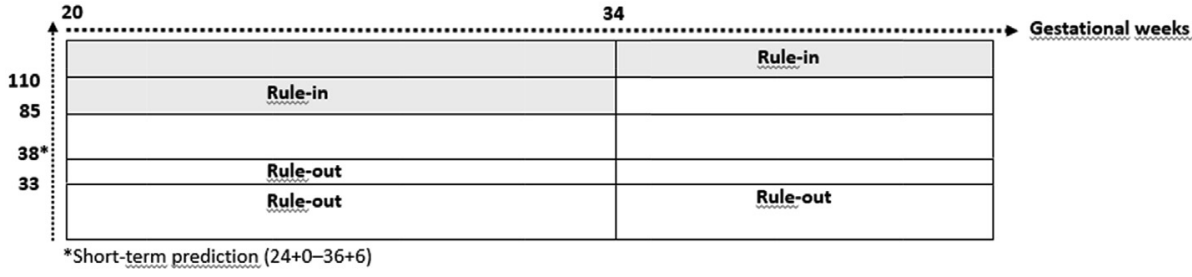

Fig. 1. The sFlt-1/PlGF ratio cut-offs recommended in aid of pre-eclampsia prediction and diagnosis. this heterogeneous disorder a development of predictive tools, prevention and treatments poses a challenge.

An imbalance of maternal proangiogenic placental growth factor (PIGF) and antiangiogenic soluble fms-like tyrosine kinase 1 (sFlt-1) has been implicated in the prediction and outcomes of PE [2-4]. The sFlt-1/ PIGF ratio is elevated in patients with PE and it is better predictor of the early-onset disease (delivery $<34$ weeks of gestation) than the lateonset disease $[3,5]$. It has been suggested that syncytiotrophoblast stress contributes to the angiogenic imbalance especially in the earlyonset disease with poor placentation [6] but angiogenic markers are released also from maternal sources [7]. With accumulating evidence for the importance of angiogenic markers as a diagnostic and prognostic marker, different cut-offs have also been defined to allow an assessment of PE (Fig. $1[2,4,8,9]$ ).

In this nested case-control study we investigated first and second/third trimester levels of sFlt-1, PlGF and their ratio in addition to soluble endoglin in the Finnish Genetics of Pre-eclampsia Consortium (FINNPEC) case-control cohort. The participants were further divided into subgroups based on onset of the disease and parity. We also tested recently recommended rule-out cut-off value 33 (20 weeks to delivery), rule-in cutoffs 85 (ad 33 weeks 6 days) and 110 ( 34 weeks to delivery) for the Elecsys immunoassay sFlt-1/PIGF ratio [10]. Furthermore, we tested a cut-off for the sFlt-1/PIGF ratio that was presented recently in the PROGNOSIS study [2]. Zeisler et al. [2] derived a single cut-off value independent of the weeks of gestation; values below 38 were considered negative and were used to rule-out PE within 1 week after assessment of the ratio.

Moreover, data on pregnancy associated placental protein A (PAPP-A) and beta human chorionic gonadotrophin ( $\beta$-hCG) were measured in the first trimester biochemical screening for fetal chromosomal abnormalities.

\section{Methods}

\subsection{Design}

FINNPEC is a cross-sectional case-control multicentre study with an established nationwide clinical and DNA database on PE women and women without PE, including their partners and infants in order to identify genetic risk factors for PE. Details of the study design, methods and procedures have been described elsewhere [11]. With this study we aimed to investigate whether maternal serum concentrations of sFlt-1, PlGF, endoglin and sFlt-1/PIGF ratio available from a subset associate with PE and clinical subtypes.

\subsection{Study subjects}

Originally 1450 patients with PE and 1065 control women without PE were recruited at the 5 Finnish university hospitals. In this study, we focused on a prospective arm and on a subset of those women from whom first and second/third trimester serum samples were available. All PE women were already diagnosed at recruitment and second/third trimester serum samples were also drawn at this timepoint. All participants provided written informed consent, and the FINNPEC study protocol was approved by the coordinating Ethics Committee of the Hospital District of Helsinki and Uusimaa.

\subsubsection{Inclusion criteria}

Nulliparous or multiparous women with a singleton pregnancy were eligible for the study. PE was defined as hypertension and proteinuria occurring after 20 weeks of gestation. Hypertension was defined as systolic blood pressure $\geq 140 \mathrm{mmHg}$ and/or diastolic blood pressure $\geq 90 \mathrm{mmHg}$ and proteinuria as the urinary excretion of $\geq 0.3 \mathrm{~g}$ protein in a 24 -h specimen, or $0.3 \mathrm{~g} / \mathrm{l}$, or two $\geq 1+$ readings on dipstick in a random urine determination with no evidence of the urinary tract infection. Each diagnosis was ascertained based on hospital records and confirmed independently by a research nurse and a study physician.

\subsubsection{Exclusion criteria}

Exclusion criteria were multiple pregnancy, maternal age less than 18 years and inability to provide an informed consent based on information in Finnish or Swedish.

\subsection{Background, obstetric and perinatal data}

Extensive information on pregnancy complications, pregnancy outcome, proteinuria, blood pressure, laboratory measurements, delivery and baby was obtained from the hospital records and maternity cards. Information on PE in previous pregnancies was verified from the hospital records.

PE was defined as early-onset when delivery occurred before $34+0$ weeks of gestation and late-onset when at $34+0$ weeks of gestation or later. Birth weights below $-2.0 \mathrm{SD}$ units were classified as small-for-gestational age (SGA) according to Finnish standards [12].

Hemolysis, elevated liver enzymes, and low platelet count (HELLP) syndrome was diagnosed when at least 2 of the following criteria were met: lactate dehydrogenase (LD) $\geq 235 \mathrm{U} / \mathrm{l}$, alanine aminotransferase $($ ALAT) $\geq 70 \mathrm{U} / 1$, aspartate aminotransferase (ASAT) $\geq 70 \mathrm{U} / 1$, and thrombocytes $\leq 100 \mathrm{E} 9 / 1$.

\subsection{Serum samples and angiogenic markers}

First and second/third trimester serum samples were collected from a subcohort from the Hospital District of Helsinki and Uusimaa. First trimester serum samples were obtained via first trimester biochemical screening for fetal chromosome abnormalities (range 9-15 weeks of gestation), and during the second and third trimesters (range 20-42 weeks of gestation) serum samples were collected at hospitals. The results on angiogenic markers measured from samples obtained during the second and third trimesters were further divided into early/ late based on the timing of the blood sampling (early: 20-33 + 6 weeks of gestation and late sample $\geq 34$ weeks of gestation).

Maternal serum sFlt-1 and PlGF concentrations were measured using sFlt-1 and PlGF electro-chemiluminescence immunoassays (ECLIA; Roche Diagnostics GmbH, Mannheim, Germany) on cobas e 601 analyzer (Hitachi High Technology Co, Tokyo, Japan). Serum concentration of endoglin (CD105) was measured using human Quantikine Endoglin ELISA kit (R\&D Systems, UK) according to manufacturer's instructions.

Pregnancy associated placental protein A (PAPP-A) and beta human chorionic gonadotrophin ( $\beta$-hCG) were analyzed by time-resolved fluoroimmunoassay according to manufacturer's instructions (PerkinElmer, Wallac, Turku, Finland). 


\subsection{Statistical analysis}

Statistical tests were performed with IBM SPSS Statistics version 24 . The normality of variable distributions was verified with the Kolmogorov-Smirnov test. Logarithmic transformation was used when appropriate. Each biomarker was ln-transformed to correct for rightskewness, and estimated means were back-transformed as geometric means and $95 \%$ confidence intervals for purposes of presentation. For the continuous variables, comparisons between groups were analysed with general linear model univariate ANOVA at baseline and with linear mixed models during the pregnancy. Selected co-variables [parity, maternal age, smoking status, body mass index (BMI), gestational weeks at sampling] were included in the models as covariates. Normality was assessed by plotting the residuals.

For the categorical variables, the comparisons were performed with the Fisher's exact test. With skewed distributions, comparisons between continuous variables were performed by the Mann Whitney $U$ test.

\section{Results}

As published earlier for the whole FINNPEC population [11], in this subcohort the PE women had higher BMI and more frequently certain preexisting medical conditions (e.g. chronic hypertension, pregestational diabetes) and gestational diabetes than controls (Table 1). The proportion of primiparous women was also higher in the PE group (Table 1). At first trimester screening, both PAPP-A and $\beta$-hCG concentrations were lower in the PE group compared to the control group.
Serum concentrations of angiogenic markers are presented in Table 2. In all timepoints PlGF, endoglin and the sFlt-1/PlGF ratio were increased in the PE group compared to the control group. The serum concentrations of sFlt-1 was increased only at second/third trimester in PE women compared to non-PE women. The proportion of women exceeding all cut-offs of the sFlt-1/PlGF ratio ( $\geq 33, \geq 38, \geq 85$ and $\geq 110$ ) was greater in the PE group (Table 2). However, there were also preeclamptic women who met NICE rule-out cut-off 33 at second/third trimester $(\mathrm{n}=10)$ or did not meet rule-in cut-off 85 (ad 33 weeks 6 days) $(n=8)$ and $110(n=4)(34$ weeks to delivery). Ten PE women who did not exceed rule-out cut-off 33 had higher prepregnancy BMI, smoked more before pregnancy, suffered more from renal disease, had more often a history of PE and the relative birth weight of the newborn was higher (Table 3a). Moreover, eight PE women did not exceed cut-off 85. The basic characteristics of these women are presented in Table 3b. Also these PE women had a trend for higher prepregnancy BMI and the relative birth weight of the newborn was higher. There were four control women who exceeded the cut-off 110 and the comparison of these women with or without exceeding cut-off 110 is presented in Table 3c. These four women suffered more from placental insufficiency and had a trend for having SGA babies. Three of these control women exceeded the rule-in cut-off already before 34 weeks of gestation.

There where altogether $49 \mathrm{PE}$ women of whom serum samples were available one week before the delivery. Of those women, only one woman did not exceed cut-off 38. In addition, there were four control women who exceeded cut-off 38 within a week of the delivery. Two of these women had gestational hypertension and SGA baby.

Table 1

Maternal and perinatal characteristics of pre-eclamptic and control groups in a subset of the FINNPEC women.

\begin{tabular}{|c|c|c|c|c|}
\hline Maternal or Perinatal Characteristics & $\begin{array}{l}\text { Pre-eclampsia } \\
(\mathrm{n}=221)\end{array}$ & $\begin{array}{l}\text { Control } \\
(n=239)\end{array}$ & $p^{*}$ & $p^{* * *}$ \\
\hline Age at delivery (y) & $30.3 \pm 5.3$ & $30.5 \pm 4.8$ & $0.495^{* * *}$ & $0.661^{\mathrm{b}}$ \\
\hline Nulliparous, n (\%) & $166(75.1 \%)$ & $137(57.3 \%)$ & $<0.001$ & \\
\hline $\mathrm{BMI}, \mathrm{kg} / \mathrm{m}^{2}$ (self-reported, pre-pregnancy) & $25.5 \pm 5.1$ & $23.9 \pm 3.9$ & $0.005^{* * * * k}$ & $<0.001^{\mathrm{c}}$ \\
\hline PAPP-A $(\mathrm{mU} / \mathrm{l})^{\mathrm{j}}$ & $1455 \pm 1193(214)$ & $1952 \pm 1209(236)$ & $<0.001^{* * * *}$ & $<0.001^{\mathrm{a}}$ \\
\hline Beta hCG $(\mu \mathrm{g} / 1)^{\mathrm{j}}$ & $45.3 \pm 30.1(214)$ & $54.4 \pm 40.3(236)$ & 0.038 & $0.005^{\mathrm{a}}$ \\
\hline Highest systolic blood pressure $(\mathrm{mmHg})^{\mathrm{h}}$ & $165 \pm 17$ & $127 \pm 14$ & $<0.001^{* * * *}$ & $<0.001$ \\
\hline Highest diastolic blood pressure $(\mathrm{mmHg})$ & $110 \pm 9$ & $85 \pm 10$ & $<0.001^{\text {*****}}$ & $<0.001$ \\
\hline Proteinuria (max.) $(\mathrm{g} / 24 \mathrm{~h})$ & $3.7 \pm 3.2(211)$ & - & - & \\
\hline Smoking before pregnancy & $58(26.9 \%)(216)$ & $57(23.9 \%)(238)$ & 0.003 & \\
\hline Smoking during pregnancy & $24(11.0 \%)(218)$ & $20(8.4 \%)(238)$ & 0.005 & \\
\hline Chronic hypertension ${ }^{\mathrm{f}}$ & $33(14.9 \%)$ & $8(3.3 \%)$ & $<0.001$ & \\
\hline Gestational hypertension $^{g}$ & - & $21(8.8 \%)$ & - & \\
\hline Gestational diabetes mellitus & $28(12.7 \%)$ & $13(5.4 \%)$ & 0.007 & \\
\hline Pregestational diabetes mellitus & $12(5.4 \%)$ & $1(0.4 \%)$ & 0.001 & \\
\hline Type 1 diabetes & $11(5.0 \%)$ & $1(0.4 \%)$ & & \\
\hline Type 2 diabetes & $1(0.5 \%)$ & - & & \\
\hline Mode of delivery & & & $<0.001$ & \\
\hline Vaginal & $133(60.2 \%)$ & $197(82.7 \%)$ & & \\
\hline Caesarean section & $88(39.8 \%)$ & $41(17.3 \%)(238)$ & & \\
\hline Gestational weeks at delivery & $36.8 \pm 3.3$ & $39.3 \pm 2.6$ & $<0.001$ & \\
\hline Birth weight, $g$ & $2792 \pm 851$ & $3476 \pm 717$ & $<0.001^{\text {k***k}}$ & $0.001^{\mathrm{e}}$ \\
\hline Relative birth weight (SD) & $-0.9 \pm 1.3$ & $-0.2 \pm 1.2$ & $<0.001$ & $0.003^{\mathrm{e}}$ \\
\hline Fetal sex, female/male (\%) & $52.9 \% / 47.1 \%$ & $55.6 \% / 44.4 \%$ & 0.042 & \\
\hline SGA & $35(15.8 \%)$ & $18(7.5 \%)$ & 0.005 & \\
\hline
\end{tabular}

"Unadjusted; "*adjusted; "**** non-parametric tests used.

() Number of available information/samples unless from all.

Data are presented as mean \pm S.D or percentages.

$\mathrm{BMI}=$ body mass index, PAPP-A = placental protein A, $\beta$-hCG $=$ beta human chorionic gonadotrophin, SGA small-for-gestational age

${ }^{\mathrm{d}}$ Adjusted for parity, mother's age at birth, prepregnancy BMI.

${ }^{\mathrm{i}}$ Based on weight and height before pregnancy, self-reported at first antenatal visit.

a Adjusted for gestational weeks at sampling.

b Adjusted for parity.

${ }^{c}$ Adjusted for parity, mother's age at birth.

e Adjusted for parity, mother's age at birth, gestation weeks, prepregnancy BMI, chronic hypertension, gestational diabetes, pregestational diabetes mellitus.

${ }^{f}$ Systolic blood pressure $\geq 140 \mathrm{mmHg}$ and/or diastolic blood pressure $\geq 90 \mathrm{mmHg}$ detected before 20 weeks of gestation.

${ }^{g}$ Blood pressure $\geq 140 / 90$, no proteinuria.

${ }^{\mathrm{h}}$ When highest diastolic value recorded.

${ }^{\mathrm{j}}$ PAPP-A and beta hCG samples were obtained at 9-15 weeks of gestation. 
Table 2

Concentrations of angiogenic markers in pre-eclamptic and control women of FINNPEC, geometric mean (95\% CI).

\begin{tabular}{|c|c|c|c|c|}
\hline & $\begin{array}{l}\text { Pre-eclampsia } \\
\text { I trimester } \mathrm{n}=221 \\
\text { II/III trimester } \mathrm{n}=175 \text { Early sample } \\
(20-33+6), \mathrm{n}=48 \text {, Late sample }(>34), \mathrm{n}=127\end{array}$ & $\begin{array}{l}\text { Control } \\
\text { I trimester } \mathrm{n}=239 \\
\text { II/III trimester } \mathrm{n}=55 \text { Early sample } \\
(20-33+6), \mathrm{n}=9 \text {, Late sample }(\geq 34), \mathrm{n}=46\end{array}$ & p unadj. & $p^{*} a d j$. \\
\hline \multicolumn{5}{|l|}{$s F l t-1(p g / m l)$} \\
\hline I trimester & $1303.9(1232.3-1379.5)(n=220)$ & $1388.1(1322.0-1457.4)$ & 0.097 & 0.349 \\
\hline II-III trimester & $10356.8(9403.2-11406.1)(\mathrm{n}=175)$ & $4278.1(3621.7-5053.8)$ & $<0.001$ & $<0.001$ \\
\hline \multicolumn{5}{|l|}{$P l G F(p g / m l)$} \\
\hline I trimester & $31.3(29.5-33.2)(n=220)$ & $41.8(39.8-43.9)$ & $<0.001$ & $<0.001$ \\
\hline II-III trimester & $81.0(73.5-89.4)$ & $164.6(130.7-207.3)$ & $<0.001$ & $<0.001$ \\
\hline \multicolumn{5}{|l|}{ Endoglin $(\mathrm{ng} / \mathrm{ml})$} \\
\hline I trimester & $5.9(5.7-6.1)(\mathrm{n}=220)$ & $5.6(5.4-5.8)$ & 0.035 & 0.005 \\
\hline II-III trimester & $41.7(37.7-46.2)$ & $16.6(14.0-19.6)$ & $<0.001$ & $<0.001$ \\
\hline \multicolumn{5}{|l|}{ sFlt-1/PlGF } \\
\hline I trimester & $42(39-45)(n=220)$ & $33(31-35)$ & $<0.001$ & $<0.001$ \\
\hline II-III trimester & $188 \pm 180^{\mathrm{b}}$ & $45 \pm 47^{b}$ & $<0.001^{* * * *}$ & na \\
\hline \multicolumn{5}{|l|}{ sFlt- $1 /$ PlGF ratio, $\geq 33$} \\
\hline II-III trimester & $165(94.3 \%)$ & $22(40.0 \%)$ & $<0.001^{c}$ & $<0.001^{\mathrm{a}}$ \\
\hline \multicolumn{5}{|l|}{ sFlt- $1 /$ PlGF ratio, $\geq 38$} \\
\hline II-III trimester & $160(91.4 \%)$ & $20(36.4 \%)$ & $<0.001^{\mathrm{c}}$ & $<0.001^{a}$ \\
\hline \multicolumn{5}{|l|}{ sFlt- $1 /$ PlGF ratio, $\geq 85$ rule-in } \\
\hline II-III trimester & $123(70.3 \%)$ & $10(18.2 \%)$ & $<0.001^{c}$ & $<0.001^{\mathrm{a}}$ \\
\hline Early sample $(20-33+6)$ & $40(83.3 \%)$ & $3(37.5 \%)$ & $0.012^{\mathrm{c}}$ & $0.004^{\mathrm{a}}$ \\
\hline \multicolumn{5}{|l|}{ sFlt- $1 /$ PlGF ratio, $\geq 110$ rule-in } \\
\hline II-III trimester & $110(62.9 \%)$ & $7(12.7 \%)$ & $<0.001^{c}$ & $<0.001^{\mathrm{a}}$ \\
\hline Late sample ( $\geq 34)$ & $71(55.9 \%)$ & $4(8.7 \%)$ & $<0.001^{c}$ & $<0.001^{a}$ \\
\hline
\end{tabular}

() Number of available information/samples unless from all.

Data are presented as geometric mean $(95 \% \mathrm{CI})$ or percentages.

"Adjusted for parity, prepregnancy BMI, mother's age at birth, gestational weeks at sampling, smoking status during pregnancy.

**** Mann-Whitney $U$ test

na $=$ test not applicable

${ }^{\text {a }}$ Logistic regression adjusted for parity, prepregnancy BMI, mother's age at birth, gestational weeks at sampling, smoking status during pregnancy.

${ }^{\mathrm{b}}$ Arithmetic mean $\pm \mathrm{SD}$.

c $\chi^{2}-$ test.

There were altogether 18 women with the HELLP syndrome $(5.8 \%)$ of whom first and/or third serum samples were available. These women fulfilled also PE criteria, 7 of them had early- and 11 late-onset disease. PE women with HELLP syndrome had higher concentration of second/ third trimester sFlt-1 and sFlt-1/PlGF ratio when compared with PE women without HELLP syndrome (data not shown).

Table 3a

Characteristics of pre-eclamptic women according to the sFlt-1/PlGF ratio cut-off 33

\begin{tabular}{|c|c|c|c|}
\hline & $\begin{array}{l}\text { II-III trim sFlt-1/ } \\
\text { PlGF } \leq 33 \text { in pre- } \\
\text { eclamptic }(n=10)\end{array}$ & $\begin{array}{l}\text { II-III trim sFlt-1/ } \\
\text { PlGF > } 33 \text { in pre- } \\
\text { eclamptic }(n=165)\end{array}$ & $p^{\mathrm{a}}$ \\
\hline Age & $29.8 \pm 5.3$ & $31.2 \pm 5.9$ & 0.425 \\
\hline Prepregnancy BMI & $29.2 \pm 5.4$ & $25.3 \pm 5.6$ & 0.020 \\
\hline Parity & $2.1 \pm 2.7$ & $0.4 \pm 1.4$ & 0.001 \\
\hline Previous PE & $5(50.0 \%)$ & $23(13.9 \%)$ & 0.003 \\
\hline $\begin{array}{l}\text { Smoking before } \\
\text { pregnancy }\end{array}$ & $7(70.0 \%)$ & $37(22.4 \%)$ & 0.001 \\
\hline $\begin{array}{l}\text { Smoking during } \\
\text { pregnancy }\end{array}$ & $1(10.0 \%)$ & $12(7.3 \%)$ & 0.754 \\
\hline Placental insufficiency & $0(0.0 \%)$ & $25(15.2 \%)$ & 0.184 \\
\hline Renal disease & $4(40.0 \%)$ & $7(4.2 \%)$ & $<0.001$ \\
\hline Chronic hypertension & $3(30.0 \%)$ & $33(20.0 \%)$ & 0.447 \\
\hline Birth weight & $3445.0 \pm 775.7$ & $2546.6 \pm 877.4$ & $<0.001$ \\
\hline $\begin{array}{l}\text { Relative birth weight, } \\
\text { SD }\end{array}$ & $0.6 \pm 0.9$ & $-1.2 \pm 1.3$ & $<0.001$ \\
\hline SGA & $0(0.0 \%)$ & $42(25.5 \%)$ & 0.067 \\
\hline $\begin{array}{l}\text { Gestational weeks of } \\
\text { delivery }\end{array}$ & $37.1 \pm 3.1$ & $36.0 \pm 3.4$ & $<0.001$ \\
\hline
\end{tabular}

${ }^{\text {a }}$ Non-parametric test.

\subsection{Onset of $P E$}

Table 4 shows the comparison of angiogenic markers between women with early- or late-onset PE and controls. At the first trimester those women with early-onset disease had decreased concentrations of

Table 3b

Characteristics of pre-eclamptic women according to the sFlt-1/PIGF ratio cut-off 85 .

\begin{tabular}{|c|c|c|c|}
\hline & $\begin{array}{l}\text { II-III trim } \\
(20-33+6 \text { gwks }) \\
\text { sFlt-1/PlGF } \leq 85 \text { in } \\
\text { pre-eclamptic }(\mathrm{n}=8)\end{array}$ & $\begin{array}{l}\text { II-III trim } \\
(20-33+6 \text { gwks }) \\
\text { sFlt-1/PlGF > } 85 \text { in } \\
\text { pre-eclamptic }(n=40)\end{array}$ & $p^{\mathrm{a}}$ \\
\hline Age & $30.0 \pm 5.7$ & $31.4 \pm 6.1$ & 0.562 \\
\hline Prepregnancy BMI & $28.9 \pm 5.0$ & $25.8 \pm 5.7$ & 0.057 \\
\hline Parity & $0.6 \pm 1.4$ & $0.5 \pm 1.9$ & 0.862 \\
\hline Previous PE & $1(12.5 \%)$ & $4(10.0 \%)$ & 1.000 \\
\hline $\begin{array}{c}\text { Smoking before } \\
\text { pregnancy }\end{array}$ & $3(37.5 \%)$ & $10(25.0 \%)$ & 0.664 \\
\hline $\begin{array}{l}\text { Smoking during } \\
\text { pregnancy }\end{array}$ & $2(25.0 \%)$ & $4(10.0 \%)$ & 0.258 \\
\hline Placental insufficiency & $2(25.0 \%)$ & $18(45.0 \%)$ & 0.440 \\
\hline Renal disease & $1(12.5 \%)$ & $2(5.0 \%)$ & 0.429 \\
\hline Chronic hypertension & $2(25.0 \%)$ & $14(35.0 \%)$ & 0.701 \\
\hline Birth weight & $1976.3 \pm 723.1$ & $1469.4 \pm 544.6$ & 0.028 \\
\hline $\begin{array}{l}\text { Relative birth weight, } \\
\text { SD }\end{array}$ & $-1.0 \pm 0.9$ & $-2.1 \pm 1.0$ & 0.004 \\
\hline SGA & $1(12.5 \%)$ & $21(52.5 \%)$ & 0.055 \\
\hline $\begin{array}{l}\text { Gestational weeks of } \\
\text { delivery }\end{array}$ & $32.4 \pm 4.0$ & $31.4 \pm 2.7$ & 0.134 \\
\hline
\end{tabular}

${ }^{\text {a }}$ Non-parametric test. 
Table 3c

Characteristics of controls according to the sFlt-1/PlGF ratio cut-off 110 .

\begin{tabular}{|c|c|c|c|}
\hline & $\begin{array}{l}\text { II-III trim }(>34 \text { gwks }) \\
\text { sFlt-1/PlGF }>110 \text { in } \\
\text { controls }(n=4)\end{array}$ & $\begin{array}{l}\text { II-III trim ( }>34 \text { gwks) } \\
\text { sFlt-1/PlGF } \leq 110 \text { in } \\
\text { controls }(n=42)\end{array}$ & $p^{\mathrm{a}}$ \\
\hline Age & $33.0 \pm 9.4$ & $31.1 \pm 4.8$ & 0.395 \\
\hline Prepregnancy BMI & $26.7 \pm 3.4$ & $24.2 \pm 3.5$ & 0.160 \\
\hline Parity & $0.5 \pm 1.0$ & $0.5 \pm 0.7$ & 0.948 \\
\hline Previous PE & $0(0 \%)$ & $0(0 \%)$ & - \\
\hline $\begin{array}{c}\text { Smoking before } \\
\text { pregnancy }\end{array}$ & $2(50.0 \%)$ & $14(33.3 \%)$ & 0.602 \\
\hline $\begin{array}{c}\text { Smoking during } \\
\text { pregnancy }\end{array}$ & $0(0 \%)$ & $3(7.1 \%)$ & 0.580 \\
\hline $\begin{array}{l}\text { Placental } \\
\quad \text { insufficiency }\end{array}$ & $2(50.0 \%)$ & $2(4.8 \%)$ & 0.033 \\
\hline Renal disease & 0 & $1(2.4 \%)$ & 0.755 \\
\hline $\begin{array}{l}\text { Chronic } \\
\quad \text { hypertension }\end{array}$ & $1(25.0 \%)$ & $1(2.4 \%)$ & 0.168 \\
\hline Birth weight & $2849.0 \pm 1167.2$ & $3596.8 \pm 561.9$ & 0.315 \\
\hline $\begin{array}{l}\text { Relative birth } \\
\text { weight, SD }\end{array}$ & $-1.3 \pm 1.9$ & $0.1 \pm 1.1$ & 0.245 \\
\hline SGA & $2(50.0 \%)$ & $3(7.1 \%)$ & 0.053 \\
\hline $\begin{array}{l}\text { Gestational weeks of } \\
\text { delivery }\end{array}$ & $38.3 \pm 3.8$ & $39.6 \pm 1.7$ & 0.585 \\
\hline
\end{tabular}

${ }^{\text {a }}$ Non-parametric test.

PlGF and increased concentrations of endoglin compared to women with late-onset disease. At second/third trimester women in the earlyonset disease had highest concentrations of sFlt-1, endoglin and elevated sFlt-1/PlGF ratio. There was no difference in the proportions of women with early- or late-onset disease exceeding the cut-off values of 33 or 38 (Table 4). Both cut-offs 85 and 110 covered greater proportion of PE women with early-onset disease compared to women with lateonset disease. However, when analyses were adjusted for parity, BMI, mother's age, gestational weeks at sampling and smoking status during pregnancy, the difference was not significant.

\subsection{Parity}

Primiparous PE women had lower second/third trimester PlGF concentration and higher concentrations of sFlt-1 and endoglin compared to multiparous PE women (Table 5). Primiparous PE women had also had higher sFlt-1/PlGF ratio at the second/third trimester. Within the control group, similar differences between primi- and multiparous women were only observed for sFlt-1 and sFlt-1/PlGF ratio at the second/third trimester.

There were greater proportion of primiparous PE women exceeding cut-offs 33 and 38 at the third trimester compared to multiparous PE women. In addition, there were more primiparous women exceeding cut-offs 85 and 110 after 34 weeks of gestation than multiparous PE women. There were no similar differences within control group.

\section{Discussion}

In this study we found that primiparous pregnancies had more antiangiogenic profile during second/third trimester compared with multiparous pregnancies. Our findings also suggest that certain maternal characteristics should be taken into account when different sFlt-1/PlGF ratio cut-offs are utilized in the clinic. For instance, PE women who met NICE sFlt-1/PlGF ratio rule-out cut-off 33 at second/third trimester had higher prepregnancy BMI, smoked more before pregnancy, and had more often renal disease and history of PE.

Table 4

Concentrations of angiogenic markers in pre-eclamptic (early and late onset) and control women, geometric mean (95\% CI).

\begin{tabular}{|c|c|c|c|c|c|c|}
\hline \multirow{2}{*}{$\begin{array}{l}\text { Maternal or Perinatal } \\
\text { Characteristics }\end{array}$} & \multicolumn{2}{|l|}{ Pre-eclampsia } & \multirow{2}{*}{$\begin{array}{l}\text { p"adj. early vs. } \\
\text { late }\end{array}$} & \multirow[t]{2}{*}{ Control } & \multirow{2}{*}{$\begin{array}{l}p^{*} \text { adj .early } v s . \\
\text { control }\end{array}$} & \multirow{2}{*}{$\begin{array}{l}p^{*} \text { adj. late vs. } \\
\text { control }\end{array}$} \\
\hline & $\begin{array}{l}\text { Early onset (delivery } \leq 34 \text { weeks of } \\
\text { gestation) }\end{array}$ & Late onset & & & & \\
\hline \multicolumn{7}{|l|}{$s F l t-1(p g / m l)$} \\
\hline I trimester & 1274 (1076-1495) (33) & 1313 (1233-1392) (188) & 0.585 & $1388(1322-1457)$ & 0.234 & 0.464 \\
\hline II-III trimester & $13,227(10,509-16,548)(37)$ & 9897 (8736-10783) (138) & 0.006 & $4278(3621-5053)(n=55)$ & $<0.001$ & $<0.001$ \\
\hline \multicolumn{7}{|l|}{$P l G F(p g / m l)$} \\
\hline I trimester & $25.5(22.2-29.5)(33)$ & 32.5 (30.5-34.6) (187) & 0.002 & $41.8(39.8-43.9)$ & $<0.001$ & $<0.001$ \\
\hline II-III trimester & $47.9(36.7-62.9)(37)$ & $92.8(85.3-102.0)(138)$ & 0.097 & $\begin{array}{l}164.6(130.7-207.3) \\
(\mathrm{n}=55)\end{array}$ & 0.020 & $<0.001$ \\
\hline \multicolumn{7}{|l|}{ Endoglin $(n g / m l)$} \\
\hline I trimester & $6.6(6.0-7.3)(33)$ & $5.8(5.6-6.0)(187)$ & 0.009 & $5.6(5.4-5.8)$ & $<0.001$ & 0.046 \\
\hline II-III trimester & $64.7(48.8-86.3)(37)$ & $37.0(33.6-40.9)(138)$ & $<0.001$ & $16.6(14.0-19.6)(n=55)$ & $<0.001$ & $<0.001$ \\
\hline \multicolumn{7}{|l|}{ sFlt-1/PlGF } \\
\hline I trimester & $49(40-61)(33)$ & $40(38-43)(187)$ & 0.031 & $33(31-35)$ & $<0.001$ & $<0.001$ \\
\hline II-III trimester & $375 \pm 271(37)^{\mathrm{b}}$ & $137 \pm 100(138)^{b}$ & $\begin{array}{l}\text { (test not } \\
\text { available) }\end{array}$ & $45 \pm 47(n=55)^{b}$ & na & na \\
\hline \multicolumn{7}{|l|}{ sFlt- $1 /$ PlGF ratio, $\geq 33$} \\
\hline II-III trimester & $36(97.3 \%)$ & $129(93.5 \%)$ & $0.744^{\mathrm{a}}$ & $22(40.0 \%)$ & $0.092^{\mathrm{a}}$ & $<0.001^{\mathrm{a}}$ \\
\hline \multicolumn{7}{|l|}{ sFlt- $1 /$ PlGF ratio, $\geq 38$} \\
\hline II-III trimester & $36(97.3 \%)$ & $124(89.9 \%)$ & $0.371^{\mathrm{a}}$ & $20(36.4 \%)$ & $0.010^{\mathrm{a}}$ & $<\mathbf{0 . 0 0 1}^{\mathrm{a}}$ \\
\hline \multicolumn{7}{|l|}{ sFlt- $1 /$ PlGF ratio, $\geq 85$} \\
\hline II-III trimester & $33(89.2 \%)$ & $90(65.2 \%)$ & $0.069^{\mathrm{a}}$ & $10(18.2 \%)$ & $0.001^{\mathrm{a}}$ & $<0.001^{\mathrm{a}}$ \\
\hline Early sample & $33(89.2 \%)$ & $7(63.6 \%)$ & $0.196^{\mathrm{a}}$ & $3(37.5 \%)$ & $0.996^{\mathrm{a}}$ & $0.064^{\mathrm{a}}$ \\
\hline \multicolumn{7}{|l|}{ sFlt- $1 /$ PlGF ratio, $\geq 110$} \\
\hline II-III trimester & $33(89.2 \%)$ & 77 (55.8\%) & $0.182^{\mathrm{a}}$ & $7(12.7 \%)$ & $<0.001$ & $<0.001^{\mathrm{a}}$ \\
\hline Late sample & - & $71(55.9 \%)$ & - & $4(8.7 \%)$ & - & $<0.001^{\mathrm{a}}$ \\
\hline
\end{tabular}

${ }^{* *}$ Adjusted for parity, prepregnancy BMI, mother's age at birth, gestational weeks at sampling, smoking status during pregnancy.

na $=$ test not applicable.

${ }^{\mathrm{c}} \chi^{2}$ - test; ${ }^{\mathrm{d}}$ Mann-Whitney $U$ test.

${ }^{\text {a }}$ Logistic regression adjusted for parity, prepregnancy BMI, mother's age at birth, gestational weeks at sampling, smoking status during pregnancy.

b Arithmetic mean \pm SD. 
Table 5

Concentrations of angiogenic markers in pre-eclamptic and control women according to the parity status, geometric mean (95\% CI).

\begin{tabular}{|c|c|c|c|c|c|c|c|c|}
\hline \multirow{2}{*}{$\begin{array}{l}\text { Maternal or } \\
\text { Perinatal } \\
\text { Characteristics }\end{array}$} & \multicolumn{2}{|l|}{ Pre-eclampsia } & \multirow{2}{*}{$\begin{array}{l}p^{*} \text { (primi vs. } \\
\text { multiparas) }\end{array}$} & \multicolumn{2}{|l|}{ Control } & \multirow{2}{*}{$\begin{array}{l}p^{*} \text { (primi vs. } \\
\text { multiparas) }\end{array}$} & \multirow{2}{*}{$\begin{array}{l}p^{*} \text { for } P E \\
\text { primipara } v s . \\
\text { control } \\
\text { primipara }\end{array}$} & \multirow{2}{*}{$\begin{array}{l}p^{*} \text { for } P E \\
\text { multipara } v s \\
\text { control } \\
\text { multipara }\end{array}$} \\
\hline & Primipara & Multipara & & Primipara & Multipara & & & \\
\hline \multicolumn{9}{|l|}{$s F l t-1(p g / m l)$} \\
\hline I trimester & $\begin{array}{l}1318.0(1235.3-1406.1) \\
(166)\end{array}$ & $\begin{array}{l}1262.1 \\
(1121.3-1420.6) \\
(55)\end{array}$ & 0.304 & $\begin{array}{l}1392.1 \\
(1299.2-1491.7) \\
(137)\end{array}$ & $\begin{array}{l}1382.6(1291.9- \\
1479.7)(102)\end{array}$ & 0.484 & 0.429 & 0.338 \\
\hline II-III trimester & $\begin{array}{l}11,610.9 \\
(10,527.0-12,807.7) \\
(128)\end{array}$ & $\begin{array}{l}7585.6 \\
(6065.1-9488.2) \\
(47)\end{array}$ & 0.001 & $\begin{array}{l}5023.6 \\
(4044.5-6239.2) \\
(35)\end{array}$ & $\begin{array}{l}3229.9 \\
(2572.9-4044.5) \\
(20)\end{array}$ & 0.005 & $<0.001$ & $<0.001$ \\
\hline \multicolumn{9}{|l|}{$P l G F(p g / m l)$} \\
\hline I trimester & $30.7(28.6-32.8)(166)$ & $\begin{array}{l}33.5(29.9-37.5) \\
(54)\end{array}$ & 0.691 & $\begin{array}{l}41.1(38.4-43.9) \\
(137)\end{array}$ & $\begin{array}{l}42.8(39.7-46.1) \\
(102)\end{array}$ & 0.852 & $<0.001$ & 0.001 \\
\hline II-III trimester & $75.4(67.2-84.7)(128)$ & $\begin{array}{l}98.5(82.5-117.6) \\
(47)\end{array}$ & 0.015 & $\begin{array}{l}147.8 \\
(118.2-185.0)(35)\end{array}$ & $\begin{array}{l}198.6 \\
(117.7-335.2)(20)\end{array}$ & 0.097 & $<0.001$ & 0.033 \\
\hline \multicolumn{9}{|l|}{ Endoglin $(n g / m l)$} \\
\hline I trimester & $6.0(5.7-6.2)(166)$ & $5.8(5.3-6.3)(54)$ & 0.619 & $5.7(5.4-5.9)(137)$ & $5.5(5.2-5.8)(102)$ & 0.098 & 0.047 & 0.066 \\
\hline II-III trimester & $47.6(42.8-53.1)(128)$ & $\begin{array}{l}29.1(23.4-36.1) \\
(47)\end{array}$ & $<0.001$ & $\begin{array}{l}18.5(14.8-23.2) \\
(35)\end{array}$ & $\begin{array}{l}13.6(10.8-23.2) \\
(20)\end{array}$ & 0.063 & $<0.001$ & $<0.001$ \\
\hline \multicolumn{9}{|l|}{ sFlt-1/PlGF } \\
\hline I trimester & $43(40-44)(166)$ & $38(33-44)(54)$ & 0.291 & 34 (31-37) (137) & $32(30-35)(102)$ & 0.445 & $<0.001$ & 0.042 \\
\hline II-III trimester & $206 \pm 184^{\mathrm{b}}(128)$ & $138 \pm 160^{\mathrm{b}}(47)$ & $<0.001^{\text {*** }}$ & $51 \pm 47^{\mathrm{b}}(35)$ & $36 \pm 47^{\mathrm{b}}(20)$ & $0.022^{k * k}$ & $<0.001$ & $<0.001$ \\
\hline \multicolumn{9}{|c|}{ sFlt- $1 /$ PlGF ratio $\geq 33$} \\
\hline II-III trimester & $126(98.4 \%)$ & $39(83.0 \%)$ & $0.001^{\mathrm{a}}$ & $16(45.7 \%)$ & $6(30.0 \%)$ & $0.179^{\mathrm{a}}$ & $<0.001^{\mathrm{a}}$ & $0.004^{\mathrm{a}}$ \\
\hline \multicolumn{9}{|c|}{ sFlt- $1 /$ PlGF ratio $\geq 38$} \\
\hline II-III trimester & $126(98.4 \%)$ & $34(72.3 \%)$ & $<0.001^{\mathrm{a}}$ & $14(40.0 \%)$ & $6(30.0 \%)$ & $0.295^{\mathrm{a}}$ & $<0.001^{\mathrm{a}}$ & $0.048^{\mathrm{a}}$ \\
\hline \multicolumn{9}{|c|}{ sFlt- $1 /$ PlGF ratio, $\geq 85$} \\
\hline II-III trimester & $100(78.1 \%)$ & $23(48.9 \%)$ & $0.001^{\mathrm{a}}$ & $7(20.0 \%)$ & $3(15.0 \%)$ & $0.602^{\mathrm{a}}$ & $<0.001^{\mathrm{a}}$ & $0.115^{\mathrm{a}}$ \\
\hline Early sample & $32(84.2 \%)$ & $8(80.0 \%)$ & $0.377^{\mathrm{a}}$ & $2(28.6 \%)$ & $1(100 \%)$ & $0.375^{\mathrm{c}}$ & $0.004^{\mathrm{a}}$ & $1.000^{c}$ \\
\hline \multicolumn{9}{|c|}{ sFlt-1/PlGF ratio, $\geq 110$} \\
\hline II-III trimester & $92(71.9 \%)$ & $18(38.3 \%)$ & $<0.001^{\mathrm{a}}$ & $5(14.3 \%)$ & $2(10.0 \%)$ & $0.337^{\mathrm{a}}$ & $<0.001^{\mathrm{a}}$ & $0.183^{\mathrm{a}}$ \\
\hline Late sample & $61(67.8 \%)$ & $10(27.0 \%)$ & $<0.001^{\mathrm{a}}$ & $3(11.1 \%)$ & $1(5.3 \%)$ & $0.448^{\mathrm{c}}$ & $<0.001^{\mathrm{a}}$ & $0.077^{c}$ \\
\hline
\end{tabular}

"Adjusted for prepregnancy BMI, mother's age at birth, gestational weeks at sampling and smoking status during pregnancy.

"*nadjusted.

${ }^{\text {a }}$ Logistic regression adjusted for prepregnancy BMI, mother's age at birth, gestational weeks at sampling and smoking status during pregnancy.

b Arithmetic mean \pm SD.

${ }^{c} \chi^{2}-$ test.

In general, our results support the increasing evidence that the pathogenesis of PE involves an imbalance between angiogenic and antiangiogenic markers. Serum concentrations of sFlt-1, endoglin and sFlt1/PIGF ratio levels are usually found to be higher and PlGF levels lower in the PE pregnancies, but absolute levels vary markedly between studies $[2,12,13]$. However, these factors have also limitations as PE biomarkers, especially for prediction and diagnosis of PE at term [6].

Maternal $\beta$-hCG and PAPP-A have also gained acceptance as potential predictors of PE at the first trimester [14]. In the current study, we were able to confirm all these previously observed differences in angiogenic markers except in higher sFlt-1 concentration at first trimester.

\subsection{Onset of $P E$}

In line with the previous literature [15] we found increased serum endoglin at second/third trimester to be associated with early-onset disease. However, to our knowledge, this is the first study to show increased serum endoglin concentration in the early-onset PE already at first trimester. At the second/third trimester sFlt-1 concentration and sFlt1/PlGF ratio were also higher in PE women with early-onset disease compared to the late-onset group. Furthermore, serum PlGF concentration was lower at the first trimester in women who later developed early-onset PE compared to women who had late-onset PE and compared to controls. PlGF is thought to exert a direct pro-angiogenic activity, and it has been demonstrated that the levels of PlGF are decreased early in pregnancies later complicated by PE [16]. Khalil et al.
[17] have also shown that maternal serum PIGF is a useful marker for PE from the first trimester onward, while the level of sFlt-1 is likely to have a predictive value from the second trimester onward. Furthermore, it has been demonstrated that serum PIGF is lower in early-onset $\mathrm{PE}$ than in late-onset PE, with no difference in serum sFlt-1 [18]. Our finding that the second/third trimester sFlt-1/PlGF ratio was higher in early-onset PE is in agreement with previous findings $[3,19,20]$. There were no differences in the number women in the early- and the late onset groups exceeding cut-offs of 33 or 38 . Accordingly, in this study sFlt-1 concentration and sFlt-1/PlGF ratio were not associated with early-onset PE at first trimester which is in contrast to some of the previous studies [21,22]. Whereas there were greater proportions of women in the early- onset group exceeding cut-offs 85 and 110. These finding highlight the theory that early- and late-onset PE have different pathophysiologic pathways. In late-onset PE there is a broader spectrum of involved mechanisms possibly due to underlying maternal conditions and endothelial injury whereas early-onset PE is more closely related to placental dysfunction and angiogenic imbalance [23-25].

Early-onset PE is also associated with higher perinatal and maternal morbidity and mortality than late-onset disease, mainly due to gestational age at delivery [26]. However, very recently Christensen [27] showed that particularly gestational age at PE onset including the earlyonset/late-onset distinction was associated with subclinical atherosclerosis 12 years after delivery. However, more studies on the predictive role of angiogenic markers in augmented risk for future cardiovascular disease are warranted. 
The use of angiogenic markers in the first-trimester prediction of early-onset PE would be clinically relevant since low-dose aspirin started before 16 weeks' gestation may be effective in the prevention of the disease or postponing the onset of the disease [28].

\subsection{Parity}

Nulliparity is a well-known risk factor for PE with a reported incidence of up to 2-3 times higher than in multiparous pregnancies [29]. The mechanisms explaining this epidemiological observation have been postulated to involve e.g. immune maladaptation and greater insulin resistance [30]. There are relatively few studies on the association between parity and circulating angiogenic markers. Higher sFlt1 levels have been reported in the first and second trimester of nulliparous women [31,32]. Furthermore, Bdolah et al. [33] have shown that nulliparous women have higher circulating sFlt1 concentration and sFlt1/PlGF ratio than multiparous women during the late third trimester. Consistent with Bdolah et al. [33], we also demonstrated higher concentration of s-Flt1 and higher sFlt/PlGF ratio at the third trimester in primiparous PE and control women compared to multiparous women. We also observed higher levels of circulating endoglin in primiparous PE women at the third trimester. Furthermore, primiparous PE women had lower concentrations of PIGF at the third trimester compared to multiparous women. It is notable that these differences were observed in analyses adjusted for most potential confounding factors (maternal BMI, age, smoking status and gestational weeks in sampling). There were also greater proportion of primiparous $\mathrm{PE}$ women exceeding all the cut-offs at third trimester compared to multiparous PE women, but there were no differences between primi- and multiparous PE women in cut-offs 85 or 110 if sample was obtained before 34 weeks of gestation. All these findings add further support to previous speculations that altered angiogenic profile may be a potential molecular mechanism that explains the link between PE and nulliparity. More studies are needed to understand the causal mechanism behind the phenomenon.

\subsection{Cut-offs}

Although there are no formal guidelines regarding the use of the sFlt-1/PlGF ratio, consensus statements have been developed by international experts on the clinical use of the Elecsys immunoassay sFlt-1/ PlGF ratio. Previously it has been shown that not all women complicated with PE have altered pro- and anti-angiogenic profile [34]. Accordingly, we observed PE women $(n=10)$ who met NICE rule-out cutoff 33 at second/third trimester. They had higher BMI, smoked more before pregnancy and suffered more from renal disease. Moreover, eight pre-eclamptic women who did not exceed cut-off 85 had a trend for higher BMI. Although the current guidelines are not based on women with clinically confirmed PE, as in our study design, we suggest that certain maternal characteristics, particularly BMI, should be taken account when different sFlt-1/PlGF ratio cut-offs are utilized in the clinic. We have recently demonstrated within the same cohort that smoking before and during pregnancy may complicate the use of angiogenic markers as a prognostic and diagnostic marker [35]. Furthermore, there are a few studies indicating that an imbalance of angiogenic markers is mild in obese pregnant women [31,36].

We conclude that the imbalance of pro- and anti-angiogenic markers is unlikely to be a primary pathophysiologic feature of PE in those women and there indeed may be angiogenic and non-angiogenic forms of $\mathrm{PE}$ as suggested earlier $[25,34,37]$. The findings that there were controls e.g. with placental insufficiency exceeding the cut-offs implicate that angiogenic imbalance is not exclusively limited to PE pregnancies. Obviously we cannot exclude the possibility that these women had developed PE later if the pregnancy had continued.

Zeisler et al. [2] have derived a cut-off value of 38 to serve as an aid in the short-term prediction of PE. They proposed this single cut-off rules out PE within one week. In our study, only one PE woman did not exceed cut-off 38 before the delivery. However, it was notable that there were four control women who exceeded cut-off 38 a week before the delivery.

\section{Strengths and limitations}

Our study has several strengths. A major strength of this study is its prospective cohort design with detailed clinical outcome information allowing to define accurately the phenotypes. In future, the combination of the angiogenic markers with clinical characteristics may substantially improve PE prediction. Detailed phenotyping enabled us to involve various adjustments for maternal characteristics and highlighted the importance of selection of clinical covariates when analysing the role of angiogenic markers in the etiology PE.

Our study has certain limitations. The sample size was limited especially when subdividing into categories. It should also be noticed that PE and control groups were not matched for gestation at sampling although this was taken account in the statistical analyses. Furthermore, the gestation at sampling for the first trimester blood samples varied from 9 to 15 weeks and this relatively wide time period might have affected e.g. the concentrations observed for sFlt-1. Moreover, there was only limited number of samples available from the second trimester. However, samples from first and second/third trimester within this study make possible to analyse changes over time.

\section{Conclusions}

In conclusion, we were able to confirm previously observed differences in angiogenic markers except in higher sFlt-1 concentration at first trimester. In future, special attention should be aimed to disentangle the role of angiogenic markers within the different maternal characteristics.

\section{Acknowledgements}

We are indebted to all the FINNPEC study participants. We appreciate the contribution of the present or former members of the FINNPEC Study Group: Tia Aalto-Viljakainen, Jenni HeikkinenEloranta, Reija Hietala, Miira Klemetti, Susanna Sainio, Terhi Saisto and Sanna Suomalainen-König (Helsinki University Hospital), Eeva Ekholm and Kaarin Mäkikallio-Anttila (Turku University Central Hospital), Marja Vääräsmäki (Oulu University Hospital), Leena Georgiadis and Leea Keski-Nisula (Kuopio University Hospital), Jukka Uotila (Tampere University Hospital), Sanna Heino, Tea Kaartokallio, Inkeri Lokki and Marja Vilkki (University of Helsinki). The expert technical assistance of Eija Kortelainen, Susanna Mehtälä, Hanna Nurmi, Aija Lähdesmäki, Satu Leminen, and Christina Salmén is gratefully acknowledged.

\section{Funding}

Funding was received from the Jane and Aatos Erkko Foundation, Päivikki ja Sakari Sohlbergin Säätiö, Academy of Finland (grants 121196, 134957, and 278941), Research Funds of the University of Helsinki, government special state subsidy for health sciences (In Finnish; Erityisvaltionosuus) at the Hospital District of Helsinki and Uusimaa, Finnish Medical Foundation, Finska Läkaresällskapet, Novo Nordisk Foundation, Finnish Foundation for Pediatric Research, Emil Aaltonen Foundation, and Sigrid Juséliuksen Säätiö.

\section{References}

[1] American College of Obstetricians and Gynecologists; Task Force on Hypertension in Pregnancy, Hypertension in pregnancy. Report of the American College of Obstetricians and Gynecologists' Task Force on Hypertension in Pregnancy, Obstet. 
Gynecol. 122 (2013) 1122-1131.

[2] H. Zeisler, E. Llurba, F. Chantraine, M. Vatish, A.C. Staff, M. Sennström, M. Olovsson, S.P. Brennecke, H. Stepan, D. Allegranza, P. Dilba, M. Schoedl, M. Hund, S. Verlohren, Predictive value of the sFlt-1:PlGF ratio in women with suspected preeclampsia, N. Engl. J. Med. 374 (2016) 13-22.

[3] S. Verlohren, I. Herraiz, O. Lapaire, D. Schlembach, M. Moertl, H. Zeisler, P. Calda, W. Holzgreve, A. Galindo, T. Engels, B. Denk, H. Stepan, The sFlt-1/PlGF ratio in different types of hypertensive pregnancy disorders and its prognostic potential in preeclamptic patients, Am. J. Obstet. Gynecol. 206 (2012) 58.e1-58.e8.

[4] S. Rana, W.T. Schnettler, C. Powe, J. Wenger, S. Salahuddin, A.S. Cerdeira, S. Verlohren, F.H. Perschel, Z. Arany, K.H. Lim, R. Thadhani, S.A. Karumanchi, Clinical characterization and outcomes of preeclampsia with normal angiogenic profile, Hypertens. Pregnancy 32 (2013) 189-201.

[5] J.P. Kusanovic, R. Romero, T. Chaiworapongsa, O. Erez, P. Mittal, E. Vaisbuch, S. Mazaki-Tovi, F. Gotsch, S.S. Edwin, R. Gomez, L. Yeo, A. Conde-Agudelo, S.S. Hassan, A prospective cohort study of the value of maternal plasma concentrations of angiogenic and anti-angiogenic factors in early pregnancy and midtrimester in the identification of patients destined to develop preeclampsia, J. Matern. Fetal Neonatal. Med. 22 (2009) 1021-1038.

[6] C.W. Redman, A.C. Staff, Preeclampsia, biomarkers, syncytiotrophoblast stress, and placental capacity, Am. J. Obstet. Gynecol. 213 (2015) S9-11.

[7] B. Huppertz, Maternal-fetal interactions, predictive markers for preeclampsia, and programming, J. Reprod. Immunol. 108 (2015) 26-32.

[8] S. Verlohren, I. Herraiz, O. Lapaire, D. Schlembach, H. Zeisler, P. Calda, J. Sabria, F. Markfeld-Erol, A. Galindo, K. Schoofs, B. Denk, H. Stepan, New gestational phasespecific cutoff values for the use of the soluble fms-like tyrosine kinase-1/placental growth factor ratio as a diagnostic test for preeclampsia, Hypertension 63 (2014) 346-352.

[9] H. Stepan, M. Hund, M. Gencay, B. Denk, C. Dinkel, W.E. Kaminski, P. Wieloch, B. Semus, T. Meloth, L.A. Dröge, S. Verlohren, A comparison of the diagnostic utility of the sFlt-1/PlGF ratio versus PlGF alone for the detection of preeclampsia/HELLP syndrome, Hypertens. Pregnancy 35 (2016) 295-305.

[10] National Institute for Health and Care Excellence (NICE), PlGF-based testing to help diagnose suspected pre-eclampsia (Triage PlGF test, Elecsys immunoassay sFlt-1/ PIGF ratio, DELFIA Xpress PIGF 1-2-3 test, and BRAHMS sFlt-1 Kryptor/BRAHMS PlGF plus Kryptor PE ratio): NICE diagnostics guidance [DG23]. https://www.nice. org.uk/guidance/dg23/chapter/1-Recommendations, 2016 (accessed May 2016).

[11] T. Jääskeläinen, S. Heinonen, E. Kajantie, J. Kere, K. Kivinen, A. Pouta, H. LaivuorifINNPEC Study Group, Cohort profile: the Finnish Genetics of Preeclampsia Consortium (FINNPEC), BMJ Open 6 (2016) e013148.

[12] J. Pihkala, T. Hakala, P. Voutilainen, K. Raivio, Characteristic of recent fetal growth curves in Finland, Duodecim 105 (1989) 1540-1546.

[13] R.J. Levine, S.E. Maynard, C. Qian, K.H. Lim, L.J. England, K.F. Yu, E.F. Schisterman, R. Thadhani, B.P. Sachs, F.H. Epstein, B.M. Sibai, V.P. Sukhatme, S.A. Karumanchi, Circulating angiogenic factors and the risk of preeclampsia, N. Engl. J. Med. 350 (2004) 672-683.

[14] L. Myatt, R.G. Clifton, J.M. Roberts, C.Y. Spong, J.C. Hauth, M.W. Varner, J.M. Thorp Jr, B.M. Mercer, A.M. Peaceman, S.M. Ramin, M.W. Carpenter, J.D. Iams, A. Sciscione, M. Harper, J.E. Tolosa, G. Saade, Y. Sorokin, G.D. AndersonEunice Kennedy Shriver National Institute of Child Health and Human Development (NICHD) Maternal-Fetal Medicine Units (MFMU) Network, First-trimester prediction of preeclampsia in nulliparous women at low risk, Obstet. Gynecol. 119 (2012) 1234-1242.

[15] R.J. Levine, C. Lam, C. Qian, K.F. Yu, S.E. Maynard, B.P. Sachs, B.M. Sibai, F.H. Epstein, R. Romero, R. Thadhani, S.A. KarumanchiCPEP Study Group, Soluble endoglin and other circulating antiangiogenic factors in preeclampsia, N. Engl. J. Med. 355 (2006) 992-1005.

[16] R. Thadhani, W.P. Mutter, M. Wolf, R.J. Levine, R.N. Taylor, V.P. Sukhatme, J. Ecker, S.A. Karumanchi, First trimester placental growth factor and soluble fmslike tyrosine kinase 1 and risk for preeclampsia, J. Clin. Endocrinol. Metab. 89 (2004) 770-775.

[17] A. Khalil, N. Maiz, R. Garcia-Mandujano, J.M. Penco, K.H. Nicolaides, Longitudinal changes in maternal serum placental growth factor and soluble fms-like tyrosine kinase- 1 in women at increased risk of pre-eclampsia, Ultrasound Obstet. Gynecol. 47 (2016) 324-331.

[18] Y.N. Kim, D.S. Lee, D.H. Jeong, M.S. Sung, K.T. Kim, The relationship of the level of circulating antiangiogenic factors to the clinical manifestations of preeclampsia, Prenat. Diagn. 29 (2009) 464-470.

[19] W. Schaarschmidt, S. Rana, H. Stepan, The course of angiogenic factors in early- vs. late-onset preeclampsia and HELLP syndrome, J Perinat. Med. 41 (2013) 511-516.

[20] C.C. Pinheiro, P. Rayol, L. Gozzani, L.M. Reis, G. Zampieri, C.B. Dias, V. Woronik, The relationship of angiogenic factors to maternal and neonatal manifestations of early-onset and late-onset preeclampsia, Prenat. Diagn. 34 (2014) 1084-1092.

[21] L.J. Vatten, A. Eskild, T.I. Nilsen, S. Jeansson, P.A. Jenum, A.C. Staff, Changes in circulating level of angiogenic factors from the first to second trimester as predictors of preeclampsia, Am. J. Obstet. Gynecol. 196 (2007) 239.e1-6.

[22] R. Romero, J.K. Nien, J. Espinoza, D. Todem, W. Fu, H. Chung, J.P. Kusanovic, F. Gotsch, O. Erez, S. Mazaki-Tovi, R. Gomez, S. Edwin, T. Chaiworapongsa, R.J. Levine, S.A. Karumanchi, A longitudinal study of angiogenic (placental growth factor) and anti-angiogenic (soluble endoglin and soluble vascular endothelial growth factor receptor-1) factors in normal pregnancy and patients destined to develop preeclampsia and deliver a small for gestational age neonate, J. Matern. Fetal. Neonatal. Med. 21 (2008) 9-23.

[23] H. Valenise, B. Vasapollo, G. Gagliardi, G.P. Novelli, Early and late preeclampsia: two different maternal hemodynamic states in the latent phase of the disease, Hypertension 52 (2008) 873-880.

[24] S.E. Maynard, S.A. Karumanchi, Angiogenic factors and preeclampsia, Semin. Nephrol. 31 (2011) 33-46.

[25] L. Myatt, J.M. Roberts, Preeclampsia: syndrome or disease? Curr. Hypertens. Rep. 17 (2015) 83.

[26] A.G. Witlin, G.R. Saade, F. Mattar, B.M. Sibai, Predictors of neonatal outcome in women with severe preeclampsia or eclampsia between 24 and 33 weeks' gestation, Am. J. Obstet. Gynecol. 182 (2000) 607-611.

[27] M. Christensen, C.S. Kronborg, R.K. Carlsen, N. Eldrup, U.B. Knudsen, Early gestational age at preeclampsia onset is associated with subclinical atherosclerosis 12 years after delivery, Acta Obstet. Gynecol. Scand. 96 (2017) 1084-1092.

[28] D.L. Rolnik, D. Wright, L.C. Poon, N. O'Gorman, A. Syngelaki, C. de Paco Matallana, R. Akolekar, S. Cicero, D. Janga, M. Singh, F.S. Molina, N. Persico, J.C. Jani, W. Plasencia, G. Papaioannou, K. Tenenbaum-Gavish, H. Meiri, S. Gizurarson, K. Maclagan, K.H. Nicolaides, Aspirin versus placebo in pregnancies at high risk for preterm preeclampsia, N. Engl. J. Med. 377 (2017) 613-622.

[29] K. Duckitt, D. Harrington, Risk factors for pre-eclampsia at antenatal booking: systematic review of controlled studies, BMJ 330 (2005) 565.

[30] Z.C. Luo, N. An, H.R. Xu, A. Larante, F. Audibert, W.D. Fraser, The effects and mechanisms of primiparity on the risk of pre-eclampsia: a systematic review, Paediatr. Perinat. Epidemiol. 21 (2007) 36-45.

[31] R.S. Mijal, C.B. Holzman, S. Rana, S.A. Karumanchi, J. Wang, A. Sikorskii, Midpregnancy levels of angiogenic markers in relation to maternal characteristics, Am. J. Obstet. Gynecol. 204 (2011) 244.e1-12.

[32] J.M. Faupel-Badger, A.C. Staff, R. Thadhani, C.E. Powe, N. Potischman, R.N. Hoover, R. Troisi, Maternal angiogenic profile in pregnancies that remain normotensive, Eur. J. Obstet. Gynecol. Reprod. Biol. 158 (2011) 189-193.

[33] Y. Bdolah, U. Elchalal, S. Natanson-Yaron, H. Yechiam, T. Bdolah-Abram, C. Greenfield, D. Goldman-Wohl, A. Milwidsky, S. Rana, S.A. Karumanchi, S. Yagel, D. Hochner-Celnikier, Relationship between nulliparity and preeclampsia may be explained by altered circulating soluble fms-like tyrosine kinase 1, Hypertens. Pregnancy 33 (2014) 250-259.

[34] R.W. Powers, J.M. Roberts, D.A. Plymire, D. Pucci, S.A. Datwyler, D.M. Laird, D.C. Sogin, A. Jeyabalan, C.A. Hubel, R.E. Gandley, Low placental growth factor across pregnancy identifies a subset of women with preterm preeclampsia: type 1 versus type 2 preeclampsia? Hypertension 60 (2012) 239-246.

[35] T. Jääskeläinen, S. Suomalainen-König, E. Hämäläinen, K. Pulkki, J. Romppanen, S. Heinonen, H. LaivuorifINNPEC, Angiogenic profile and smoking in the Finnish Genetics of Pre-Eclampsia Consortium (FINNPEC) cohort, Ann. Med. 49 (2017) 593-602.

[36] C.A. Zera, E.W. Seely, L.E. Wilkins-Haug, K.H. Lim, S.I. Parry, T.F. McElrath, The association of body mass index with serum angiogenic markers in normal and abnormal pregnancies, Am. J. Obstet. Gynecol. 211 (2014) e1-e7.

[37] A.C. Staff, S.J. Benton, P. von Dadelszen, J.M. Roberts, R.N. Taylor, R.W. Powers, D.S. Charnock-Jones, C.W. Redman, Redefining preeclampsia using placenta-derived biomarkers, Hypertension 61 (2013) 932-942. 